\title{
Serum levels of some selenodependent proteins in thyroid disorders
}

\author{
Alexandra Gabriela CATIANIS ${ }^{1}$, Bogdana VIRGOLICl ${ }^{1}$, Alexandra TOTAN ${ }^{2}$, Horia $\mathrm{VIRGOLICl}^{1}$, \\ Maria GREABU ${ }^{2}$, Maria MOHORA ${ }^{1}$ \\ ${ }^{1}$ Faculty of General Medicine, "Carol Davila" University of Medicine and Pharmacy, Bucharest, \\ Romania \\ ${ }^{2}$ Faculty of Dental Medicine, "Carol Davila" University of Medicine and Pharmacy, Bucharest, Romania
}

\begin{abstract}
Introduction. The implications of oxidative stress and inflammation are unanimously accepted in thyroid disease.

Objective. The aim of our study was to find correlations between serum antioxidant markers: glutathione peroxidase-3 (GPx-3), thioredoxin (Trx), total antioxidant capacity (TAC), inflammatory marker IL-8 and thyroid hormones, TSH and anti-thyroperoxidase antibodies (ATPO antibodies). Patients with different thyroid disorders were compared.

Materials and methods. The study included 57 patients (men and women), divided into three groups: 26 patients with hyperthyroidism, 19 patients with hypothyroidism and 12 patients with autoimmune thyroiditis. The control group was represented by 33 healthy subjects. All the participants provided the informed consent for this study.

Results. Our results showed that the patients with autoimmune thyroiditis had the highest level of Il-8 and the lowest TAC. Both Trx and GPX-3 were negatively correlated with TAC and IL-8 was positively correlated with the free T4 level, the ATPO antibodies titers, GPX-3 activity and negatively with serum TAC values.

Conclusions. Systemic oxidative stress and inflammation are present in thyroid disorders, act in cluster and potentiate each other. The presence of autoimmune antibodies worsens the biological picture.
\end{abstract}

Keywords: hyperthyroidism, hypothyroidism, autoimmune thyroiditis, antioxidants, inflammation

\section{INTRODUCTION}

Thyroid peroxidase, the enzyme involved in thyroid hormone synthesis requires $\mathrm{H}_{2} \mathrm{O}_{2}$, which is indispensable for iodine intrafollicular oxidation. The hydrogen peroxide is considered a reactive oxygen species and in the thyroid gland hyperfunction it is responsible for the increased local lipid peroxidation (1).
Also, the thyroid hormones, by stimulating the metabolic rate and the activity of the respiratory chain, generate superoxide, a reactive oxygen radical which will increase finally the systemic oxidative stress (2). The xanthine oxidase/hypoxanthine system responsible for retroocular fibroblast proliferation in Basedow-Graves disease (3), increased activities of Kupffer cells and cytochrome P-450 reductase in the liver could 
be alternative sources of reactive species in hyperthyroidism (4).

Excess thyroid-stimulating hormone (TSH) per se (5), secondary dyslipidemia and the autoimmune thyroid diseases as Hashimoto's thyroiditis, associated with low-grade chronic inflammation (6), can lead to an oxidative stress status in hypothyroidism. Both thyroid disorders, the hyperthyroidism and the hypothyroidism are associated with increased oxidative stress and inflammation. Vicious circles have been described between oxidative stress, thyroid hormones derangement and inflammation. Mancini et al. (7) supposed that in the thyroid gland, hypothyroidism can be developed by the high level of cytokines and free radicals, low antioxidant defense and hypothyroidism reinforces the oxidative stress at the tissue level, which worsens hypothyroidism by inhibiting deiodinases.

The enzymes GPx, the thioredoxin reductase Trx-R and the deiodinases, are selenocysteine enzymes and their activity depend on the availability of the trace element, selenium. These enzymes have a potential role on redox and inflammatory reactions, in autoimmune processes and in modulating the thyroid hormone levels. GPx catalyzes peroxide ( $\mathrm{ROOH})$ degradation and from the six isoforms, the GPx-3, the plasma one is responsible for the extracellular defence. Trx- $R$ is a sensor for cellular reactive oxygen species and via transcription factors AP-1, NF-kB and p53 can regulate gene expression (8). Trx-R catalyzes the reaction for Trx recovery, by using NADPH. Thioredoxin acts as a specific electron donor for peroxidases (9).

IL-8 was proposed as an universal marker of inflammation (10). It is an important activator and chemoattractant for neutrophils, and has been implicated in a variety of inflammatory diseases, including thyroid diseases (11). Elevated circulating IL-8 in hyperthyroidism and deregulated production of IL- 8 in autoimmune thyroid diseases were observed $(11,12)$. It was demonstrated that interleukin (like IL-6) induced reactive oxygen production enhances GPx and Trx-R activities (13).

Data literature suggests that selenium supplementation of patients with autoimmune thyroiditis is associated with a reduction in anti-thyroperoxidase antibody levels, improved thyroid ultrasound features, and improved quality of life (14).

The aim of this study was to find associations between serum inflammatory, antioxidant and autoimmune parameters in three thyroid disorders: hyperthyroidism, hypothyroidism and autoimmune thyroiditis. Knowing more about the pathogenic biological modifications in thyroid diseases we can get a better knowledge for clinical studies control and disease management.

\section{MATERIAL AND METHODS}

The study included 57 patients ( 8 men and 49 females), divided into 3 groups: 26 patients with hyperthyroidism ( 6 men and 20 females), 19 patients with hypothyroidism ( 2 men and 17 women) and 12 with autoimmune thyroidis (12 women). The control group was represented by 33 healthy euthyroid subjects (3 men and 30 women), with an average age similar to patients. All the participants, patients and controls, were from an outpatient clinic, from Bucharest. They provided the informed consent for the study, and the data was registered by the scientific Research Ethics Committee, approval no 11605/11.05.2021.

The study was a retrospective case control one. The patients enrolled were newly diagnosed cases, without any treatment. Clinical investigation and laboratory measurements (TSH, T4, FT4, FT3, ATPO) were used for thyroid dysfunction diagnosis. The patients with hyperthyroidism, hypothyroidism or autoimmune thyroid disease were included in the study. The data collected was between January 2018-December 2019 and the exclusion criteria were: intake of ethanol > $20 \mathrm{~g} /$ day, chronic inflammatory diseases, chronic treatment with cortisol, cancer, diabetes mellitus, viral or bacterial infections.

Differences between groups were analyzed using Student t-test on independent samples and correlations between parameters were evaluated by the Pearson test. A value for $p<0.05$ was considered statistically significant.

The parameters analyzed were the serum antioxidants: GPx-3 (enzyme glutathione peroxidase-3, GPx-3), thioredoxin (Trx), total antioxidant capacity (TAC), the inflammatory marker (interleukin 8, IL-8) and thyroid hormones: TSH and anti-thyroperoxidase antibodies (ATPO antibodies) and free thyroxine (freeT4, FT4).

Measurement of serum TSH, FT4 was performed using an automatic immunoassay system (IMMULITE 1000, Siemens Germany). ELISA methods were used for: TAC, Trx and GPx measurements. The ELISA kits were from $\mathrm{GmbH}$ Achen Germany with ID ABIN no. 510022 (for TAC), no. 6574192 (for Trx) and no. 6956201 (for GPx).

\section{RESULTS}

We compared serum enzymatic and nonenzymatic antioxidants, the inflammatory marker IL-8, the thyroid epiphysis control hormones (TSH and free T4) and ATPO antibodies in patients with thyroid dysfunction versus control. All the parameters had higher values in patients, with the exception of TAC which was lower (table 1). 
TABLE 1. Experimental data in control group and thyroid disorders group

\begin{tabular}{|l|c|c|c|}
\hline \multicolumn{1}{|c|}{ Parameter } & $\begin{array}{c}\text { Control group } \\
(\mathrm{n}=33)\end{array}$ & $\begin{array}{c}\text { Patients } \\
(\mathrm{n}=57)\end{array}$ & tTest $(\mathrm{p})$ \\
\hline Age(years) & $55 \pm 15$ & $54 \pm 18$ & Ns. \\
\hline TAC $(\mathrm{mmol} / \mathrm{l})$ & $59.8 \pm 14$ & $53,7 \pm 13$ & $<0.03$ \\
\hline Trx $(\mathrm{pg} / \mathrm{ml})$ & $1,288 \pm 113$ & $1,343 \pm 152$ & $<0.05$ \\
\hline GPx-3 (microU/l) & $210 \pm 37$ & $244 \pm 68$ & $<0.01$ \\
\hline IL-8 $(\mathrm{pg} / \mathrm{ml})$ & $18.5 \pm 2.6$ & $21.7 \pm 9$ & $<0.05$ \\
\hline $\begin{array}{l}\text { ATPO antibodies } \\
\text { IU/ml }\end{array}$ & $14 \pm 6$ & $152.9 \pm 69$ & $<0.001$ \\
\hline TSH $(\mu \mathrm{Ul} / \mathrm{ml})$ & $1.8 \pm 0.6$ & $4.58 \pm 2.1$ & $<0.001$ \\
\hline FT4 $(\mathrm{ng} / \mathrm{dl})$ & $1.3 \pm 0.23$ & $15.8 \pm 5.6$ & $<0.001$ \\
\hline
\end{tabular}

TAC = total antioxidant capacity, $T r x=$ thyoredoxine, GPX-3 = glutathion peroxidase 3, IL-8 = interleukin, ATPO antibodies = anti-thyroperoxidase antibodies, $T S H=$ thyroid stimulating hormone, FT4 = free thyroxin

We also compared the three groups of patients (table 2).

TABLE 2. Experimental data in patients, the comparison of the three groups of patients

\begin{tabular}{|l|c|c|c|}
\hline Parameter & $\begin{array}{c}\text { Hyperthyroid } \\
\text { patients (n=26) } \\
\text { Group HT }\end{array}$ & $\begin{array}{c}\text { Hypothyroid } \\
\text { patients } \\
(n=19) \\
\text { Group hT }\end{array}$ & $\begin{array}{c}\text { Autoimmune } \\
\text { thyroiditis } \\
\text { patients ( } n=12) \\
\text { Group AT }\end{array}$ \\
\hline Age (years) & $50.1 \pm 18$ & $58.2 \pm 18.9$ & $52 \pm 16$ \\
\hline TAC (mmol/l) & $51.1 \pm 11.8$ & $63 \pm 11$ & $45.4 \pm 8.8$ \\
\hline Trx (pg/ml) & $1,306.5 \pm 187.9$ & $1,346 \pm 84$ & $1,421 \pm 126$ \\
\hline $\begin{array}{l}\text { GPx-3 } \\
\text { (microU/l) }\end{array}$ & $192.4 \pm 7.7$ & $208 \pm 44$ & $250 \pm 69$ \\
\hline IL-8 (pg/ml) & $16,6 \pm 3,8$ & $17 \pm 2.6$ & $34.4 \pm 11.3$ \\
\hline $\begin{array}{l}\text { ATPO } \\
\text { antibodies } \\
(\mathrm{IU} / \mathrm{ml})\end{array}$ & $101 \pm 56$ & $141 \pm 98$ & $255 \pm 101$ \\
\hline TSH ( $\mu \mathrm{Ul} / \mathrm{ml})$ & $1.19 \pm 0.8$ & $10.22 \pm 5.4$ & $2.27 \pm 1.1$ \\
\hline FT4 (ng/dl) & $17.1 \pm 6.3$ & $12.8 \pm 2.3$ & $18 \pm 7.8$ \\
\hline
\end{tabular}

$T A C=$ total antioxidant capacity, $\operatorname{Tr} x=$ thyoredoxine, $G P x-3=$ glutathion peroxidase 3, IL-8 = interleukin, ATPO antibodies = anti-thyroperoxidase antibodies, TSH = thyroid stimulating hormone, FT4 = free thyroxin
tTest results:

- $\quad$ group HT vs. group hT, TAC $p<0.001 ;$ Trx Ns; GPx-3 Ns; IL-8 Ns; ATPO antibodies Ns

- $\quad$ group HT vs. group AT, TAC Ns; Trx $p<0.05$; GPx-3 p < 0.03; IL-8 p < 0.0001; ATPO antibodies $p<0.001$

- $\quad$ group hT vs. group AT, TAC $p<0.001 ;$ Trx $p<0.05$; GPx-3 p < 0.05; IL-8 $p<0.0001 ;$ ATPO antibodies $p<0.01$

In the patient group ( $n=57$ ) we calculated the simple linear correlations between the studied parameters and we observed that the serum TAC values were negatively correlated with GPx-3 activity $(r=-0.52)$ (Figure 1 ) and with serum Trx concentration ( $r=-0.48$ ) (Figure 2).

A positive, strong association between serum Trx and GPx-3 activity was demonstrated $(r=0.79)$. Trx and GPx are stress-inducible protein and TAC can be "exhausted" in the presence of an increased oxidative stress. Observing the three groups of patients, the one with autoimmune thyroiditis had the lowest TAC and the highest level for the induced antioxidants proteins. These results reflect a high systemic oxidative stress in autoimmune thyroiditis.

An inflammatory status accompanies the oxidative stress and this is demonstrated by the increased serum IL-8 level. The IL-8 concentration was positively correlated with the free T4 level ( $r=0.35)$ (Figure 3), with GPx-3 activity ( $r=0.52$ ) (Figure 4 ) with the ATPO antibodies titers ( $r=0.37$ ) (Figure 5$)$ and negatively with serum TAC values $(r=-0.36)$.

\section{DISCUSSIONS}

Oxidative stress develops when the free radicals exceed the antioxidant defense. There are researchers who consider that in hyperthyroidism, the high production of free radicals and in hypothyroidism, the low an-

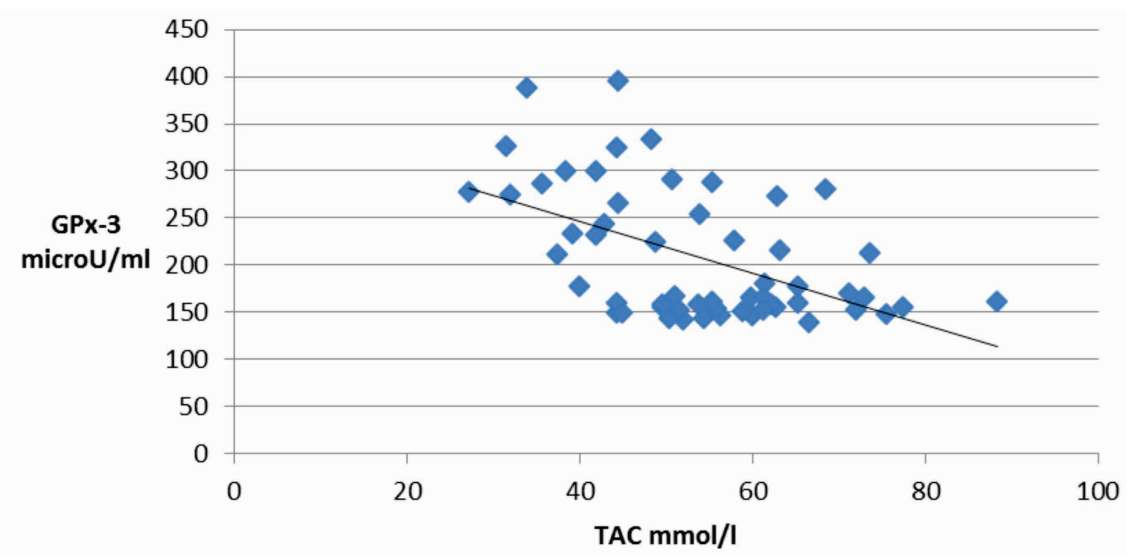

FIGURE 1. Correlation between GPX-3 activity and TAC concentration for all patients $(N=57)$ 

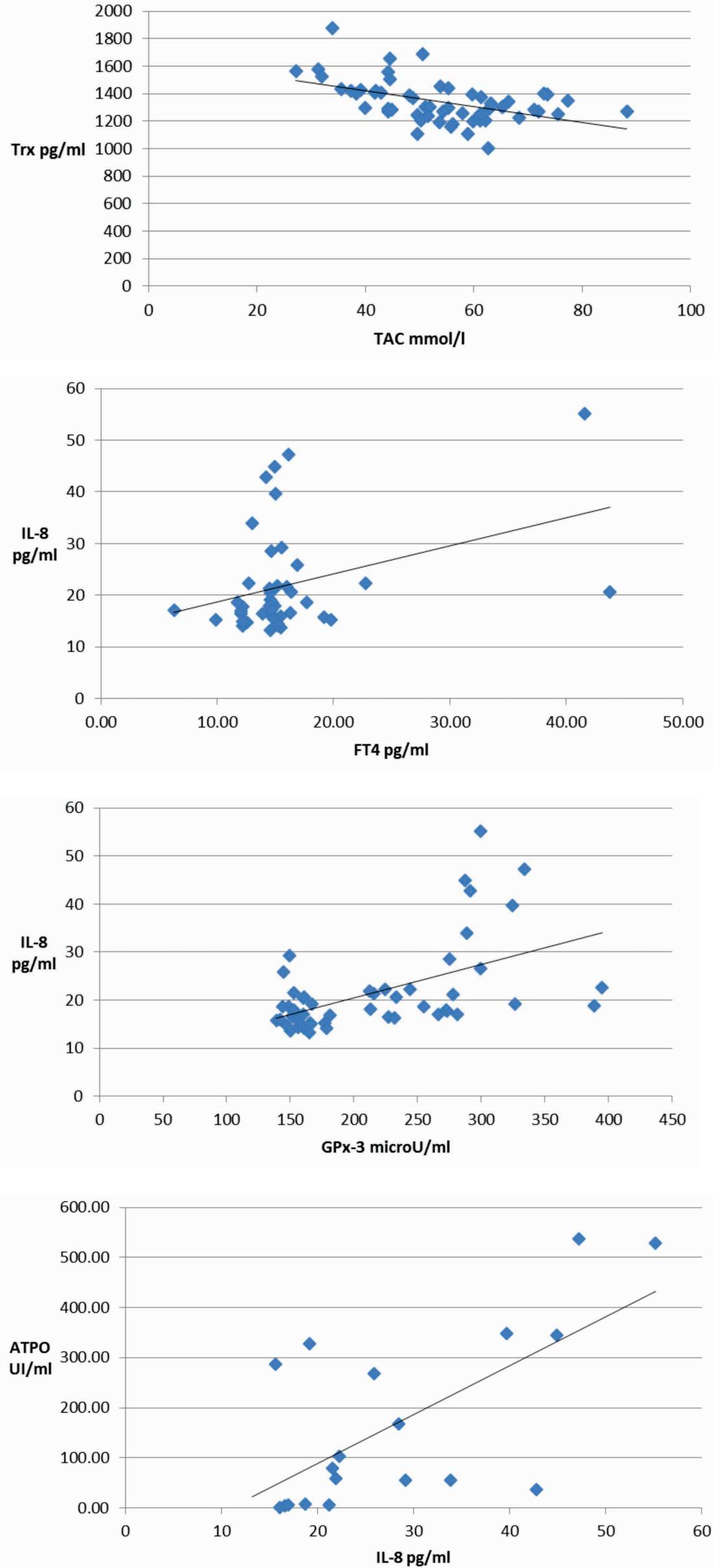

FIGURE 2. Correlation between Trx and TAC concentration for all patients $(n=57)$

FIGURE 3. Correlation between IL-8 and FT4 for all patients $(n=57)$

FIGURE 4. Correlation between IL-8 and GPX for all patients $(n=57)$

FIGURE 5. Correlation between ATPO antibodies and IL-8 
tioxidant defense can represent the two mechanisms that can cause oxidative stress in these diseases $(7,13)$.

In our study, we compared serum enzymatic and nonenzymatic antioxidants, the inflammatory marker IL-8, the thyroid epiphysis control hormones (TSH and free T4) and ATPO antibodies in patients with thyroid dysfunction versus control. All the parameters had higher values in patients, with the exception of TAC which was lower than in controls.

In clinical thyroid dysfunction studies, higher serum levels for IL-8 were demonstrated and it seems that this interleukin has a role in the pathogenesis of thyroid diseases $(15,16)$. Kobawala et al. consider that high level of IL-8 may represent a target for innovative diagnostic and therapeutic strategies (15).

In the scientific literature focused on thyroid diseases, higher serum IL-8 values are in unison, but serum TAC levels are controversial. The serum TAC measurement done in euthyroid, hyperthyroid and hyperthyroid patients $(n=93$ ) showed that the antioxidant status was not significantly altered among the different group patients (17). Other studies demonstrated that in both diseases, hyperthyroidism and hypothyroidism, serum TAC levels were significantly lower than in euthyroid subjects $(18,19)$.

Water-soluble molecules such as albumin, bilirubin, ascorbic acid, uric acid, glutathione and liposoluble molecules like vitamin $\mathrm{E}$ and coenzyme $\mathrm{Q}$ are "scavengers" molecules and contribute to the total antioxidant capacity of the serum (20). Selenium is a trace antioxidant element and it is involved in the thyroid hormons metabolism. Plasma selenium is localized mainly within two proteins, pGPx known as GPx-3 and the plasma glycoprotein SePP, known as selenoprotein P. GPx detoxifies lipid peroxides by transforming them in the corresponding alcohol (21). GPx isoforms degradate the peroxide of hydrogen and other hydroperoxides. While intracellular GPx acts on hydroperoxides of free fatty acids, the plasma GPx transforms hydroperoxides of phospholipids. It is worth mentioning that the radical overproduction in thyrotoxicosis can induce antioxidant enzymes in experimental hyperthyroidism (22).

The thioredoxin reductase-thioredoxin complex (Trx-R/Trx) acts as an intracellular defense mechanism, but the serum Trx can be measured for clinical thyroid studies. Kiharo et al. (23) demonstrated that Trx is involved in regulating production of thyroid hormones and that serum Trx is highly produced under stress, in Graves' disease $(9,23)$.

Many studies focused on serum lipid peroxidation/ total antioxidant defense imbalance, in overt hypothyroidism versus euthyroid subjects, showed high serum levels of malonyldialdehyde (MDA), a marker of increased oxidative stress $(24,25)$. Some authors pro- posed MDA as a useful biomarker to measure and monitor oxidative stress in hypothyroid patients (26). Most of these studies, but not all, found a lower serum total antioxidant capacity in hypothyroidism. Also, in autoimmune thyroiditis, the imbalance between the high serum MDA and lower TAC levels has been emphasized. The correlation of thyroid antibodies with biomarkers of oxidative stress may reflect the role of autoimmunity in the development of oxidative stress (27). Antioxidant treatments were proposed in thyroid diseases, either in hyperthyroidism or in hypothyroidism. The helpful effect of some antithyroid drugs is increased by their antioxidant effects (22). The role of the addition of antioxidant in the form of selenium in hypothyroidism has been inconclusive (26).

Our results showed that patients with autoimmune thyroiditis had important modifications of the studied parameters, the lowest TAC and highest IL-8.

As a paradox, in our study we obtained an increased TAC in hypothyroid patients versus hyperthyroid patients. The results of studies on oxidative stress and inflammation in thyroid diseases are controversial. Perhaps the relatively small number of patients enrolled in the studies, the duration and severity of the disease influence the results. However, the present study shows that if the thyroid dysfunction is associated with the presence of autoantibodies, the biological picture is greatly worsened.

The presence of ATPO antibodies in the blood suggests that the cause of thyroid disease is an autoimmune disorder. Antibodies against the thyroid gland produce chronic autoimmune thyroiditis with lymphocytic infiltration and fibroses, leading to goiter or atrophy of the thyroid gland (1).

Selenium administration, by yet unknown mechanisms, might be beneficial in systemic inflammatory response syndrome and normalized the thyroid hormone serum levels in trauma patients and preterm neonates (8). Several studies reported on the benefit of selenium treatment in autoimmune thyroid disease and the trace element improved the inflammatory status in these patients and increased the GPx activity (14).

\section{CONCLUSIONS}

Our results emphasize that in thyroid diseases, as hyperthyroidism, hypothyroidism and autoimmune thyroiditis, systemic oxidative stress and inflammation are present and act in cluster. The presence of autoimmune antibodies worsens the biological picture. The relation cause-effect of these pathological conditions with the thyroid epiphysis axis function needs more studies. 


\section{REFERENCES}

1. Andrade MA, Olarte OR. Hypothyroidism. IntechOpen, 2019.

2. Song Y, Driessens N, Costa M, et al. Roles of Hydrogen Peroxide in Thyroid Physiology and Disease. The Journal of Clinical Endocrinology \& Metabolism. 2007;92(10):3764-3773.

3. Zarković M. The role of oxidative stress on the pathogenesis of Graves' disease. J Thyroid Res. 2012;2012:302537.

4. Boisier X, Schön M, Sepúlveda A, et al. Derangement of Kupffer cell functioning and hepatotoxicity in hyperthyroid rats subjected to acute iron overload. Redox Report. 1999;4(5):243-250.

5. Nanda N, Bobby Z, Hamide A. Association of thyroid stimulating hormone and coronary lipid risk factors with lipid peroxidation in hypothyroidism. Clin Chem Lab Med. 2008:46:674-9.

6. Marchio P, Guerra-Ojeda S, Vila JM, Aldasoro M, Victor VM, Mauricio MD. Targeting Early Atherosclerosis: A Focus on Oxidative Stress and Inflammation. Oxid Med Cell Longev. 2019 Jul 1;2019:8563845.

7. Mancini A, Di Segni C, Raimondo S, et al. Thyroid Hormones, Oxidative Stress, and Inflammation. Mediators Inflamm. 2016; 2016:6757154.

8. Köhrle J, Jakob F, Contempré B, Dumont JE. Selenium, the thyroid, and the endocrine system. Endocr Rev. 2005 Dec; 26(7):944-84.

9. Nordberg J, Arnér ES. Reactive oxygen species, antioxidants, and the mammalian thioredoxin system. Free Radic Biol Med. 2001 Dec 1;31(11):1287-312.

10. Shahzad A, Knapp M, Lang I, Köhler G. Interleukin 8 (IL-8) - a universal biomarker? Int Arch Med. 2010 Jun 15;3:11.

11. Hirooka Y, Mitsuma T, Nogimori T, Ishizuki Y. Deregulated production of interleukin-8 (IL-8) in autoimmune thyroid disease studied by newly developed IL-8 radioimmunoassay. Endocr Regul. 1993 Mar;27(1):11-5.

12. van der Spek AH, Surovtseva OV, Aan S, et al. Increased circulating interleukin-8 in patients with resistance to thyroid hormone receptor a. Endocr Connect. 2017; 6(8):731-740.

13. Wajner SM, Rohenkohl HC, Serrano T, et al. Sodium selenite supplementation does not fully restore oxidative stress-induced deiodinase dysfunction: Implications for the nonthyroidal illness syndrome. Redox Biol. 2015 Dec;6:436-445.

14. Hongyan L, Chunhui Z, Hongsheng W et al. Significance of Serum IL-2, IL-6 and IL-8 Detection in Patients with Hyperthyroidism and Subclinical Hyperthyroidism. Science Journal of Public Health. 2019;7(5):159-162.

15. Kobawala TP, Patel GH, Gajjar DR, et al. Clinical utility of serum interleukin-8 and interferon-alpha in thyroid diseases. J Thyroid Res. 2011;2011:270149.

16. Sivakanesan R, Wickramarathne AMSM, Nanayakkara DKK. Serum Thyroid Stimulating Hormone and Antioxidant Status in Patients with Suspected Thyroid Dysfunction. MOJ Clinical \& Medical Case Reports, 2014;1(1).

17. Kalaivanam K, Anjaneyulu O, Santhosh KN. Total Antioxidant Capacity and Its Association with Oxidative Stress Markers in Subclinical Hypothyroidism. International Journal of Biotechnology and Biochemistry. 2019;15(1):53-58.

18. Olia JBH, Ansari MHK, Rasmi Y et al. Evaluation of Malondialdehyde Levels and Total Antioxidant Capacity in Patients with Hyperthyroidism. International Journal of Research in Applied and Basic Medical Sciences. 2019; 5(2):121-127.
19. Ventura M, Melo M, Carrilho F. Selenium and Thyroid Disease: From Pathophysiology to Treatment. Int J Endocrinol. 2017; 2017:1297658.

20. Tan BL, Norhaizan ME, Liew WP, et al. Antioxidant and Oxidative Stress: A Mutual Interplay in Age-Related Diseases. Front Pharmacol. 2018;9:1162.

21. Sarikaya E, Doğan S. Glutathione Peroxidase in Health and Diseases, Glutathione System and Oxidative Stress in Health and Disease. Intechopen, 2020.

22. Petrulea M, Muresan A, Duncea I. Oxidative Stress and Antioxidant Status in Hypo- and Hyperthyroidism, Antioxidant Enzyme. IntechOpen, 2012.

23. Kiharo M, Yamauchi A, Miyauchi A, et al. Expression of thioredoxin in patients with Graves' diseaseMay. International Journal of Molecular Medicine 2005;15(5):795-9.

24. Dumitriu L, Bartoc R, Ursu H, et al. Significance of high levels of serum malonyl dialdehyde (MDA) and ceruloplasmin (CP) in hyper- and hypothyroidism. Endocrinologie. 1988 Jan-Mar;26(1):35-38.

25. Torun ANY, Kulaxizoglu S, Kulaxizoglu M, et al. Serum total antioxidant status and lipid peroxidation marker malondialdehyde levels in overt and subclinical hypothyroidism. Clinical Endocrinology. 2008;70(3):469-74.

26. Chakrabarti SK, Ghosh S, Banerjee S, et al. Oxidative stress in hypothyroid patients and the role of antioxidant supplementation. Indian J Endocrinol Metab. 2016; 20(5):674-678.

27. Metwalley KA, Farghaly HS, Saad K, et al. Oxidative status in children and adolescents with autoimmune thyroiditis. Clin Exp Med. 2016 Nov; 16(4):571-575.

Conflict of interest: none declared Financial support: none declared 